\title{
Use of alginate gel in the pathological work-up of the endoscopically resected mucosal lesions
}

\author{
Shu Ichihara - Masaki Hasegawa - Akari Iwakoshi • \\ Tomoko Sato $\cdot$ Suzuko Moritani
}

Received: 30 October 2010 / Accepted: 23 November 2010 /Published online: 14 December 2010

(C) The Author(s) 2010. This article is published with open access at Springerlink.com

Dear Sir,

Endoscopic resection of mucosal lesions has become a common practice for early stage gastrointestinal neoplasms $[1,2]$. Consequently, endoscopically resected mucosal lesions are increasingly examined in pathology laboratories. The success of the endoscopic management of such neoplasms heavily depends on the precise and complete histological examination of the resected specimen regarding the margin status, tumor depth, histological type, and lymphovascular invasion.

Most Japanese guidelines recommend that all endoscopically resected mucosal lesions are sliced into parallel sections, each measuring $2-3 \mathrm{~mm}$ in thickness. However, the endoscopically resected mucosa is often so fragile, distorted, and irregular in thickness and consistency that the specimens easily get broken apart during slicing. Furthermore, there may be originally defects in the specimen due to technical reasons. To protect the specimen from breaking and misplacing during pathology work-up and to obtain

S. Ichihara $(\bowtie) \cdot$ M. Hasegawa $\cdot$ S. Moritani

Department of Pathology, Nagoya Medical Center,

Sannomaru 4-1-1 Naka-ku,

Nagoya, Aichi 460-0001, Japan

e-mail: shu-kkr@umin.ac.jp

\section{A. Iwakoshi}

Department of Pathology,

Nagoya University Graduate School of Medicine,

65 Tsuruma-Cho, Showa-Ku,

Nagoya, Aichi 466-8550, Japan

T. Sato

Department of Pathology, Social Insurance Chukyo Hospital,

1-1-10, Sanjo, Minami-ku,

Nagoya, Aichi 457-8510, Japan correctly oriented sequential slices, some authors have proposed embedding the whole specimen in paraffin or agar before slicing $[3,4]$. These are reasonable proposals but the problems with the use of paraffin and agar prior to slicing is that they need the control of temperature to melt and solidify and they are consequently laborious.

Here, we suggest that sodium alginate, a polysaccharide distributed widely in the cell walls of brown algae, may be an ideal material to generate a gel containing the delicate and fragile specimens. The advantage of sodium alginate is its simple gelation with divalent cations such as $\mathrm{Ca}^{2+}$ in room temperature, its cheapness, and its harmlessness. Sodium alginate sol has been used as a submucosal injection material at endoscopic submucosal resection in animal experiments [5] and at bedside in some countries.

We have found that embedding endoscopically resected mucosa in its entirety within sodium alginate gel is an efficient treatment to obtain slices of even thickness (Fig. 1). After inking the margins, we put the specimen in a plastic container filled with $2 \%$ sodium alginate liquid and add $1 \mathrm{M}$ calcium chloride to solidify. Gelation begins from the surface to the core of the alginate gel. When the thickness of the alginate sol is $6 \mathrm{~mm}$, it takes about $1 \mathrm{~h}$ for the transparent alginate gel to become gel in its entirety.

When the lesion is a polyp, the procedure is simpler. After inking the margin, we dip the entire polyp into $2 \%$ sodium alginate liquid and then drop it into $1 \mathrm{M}$ calcium chloride, resulting in a polyp coated with a layer of sodium alginate after a few minutes. The encapsulated polyp is tough enough to be cut into parallel sections by trimming knife, each measuring $2-3 \mathrm{~mm}$ in thickness to show the relationship between the neoplasm and the inked margins. 


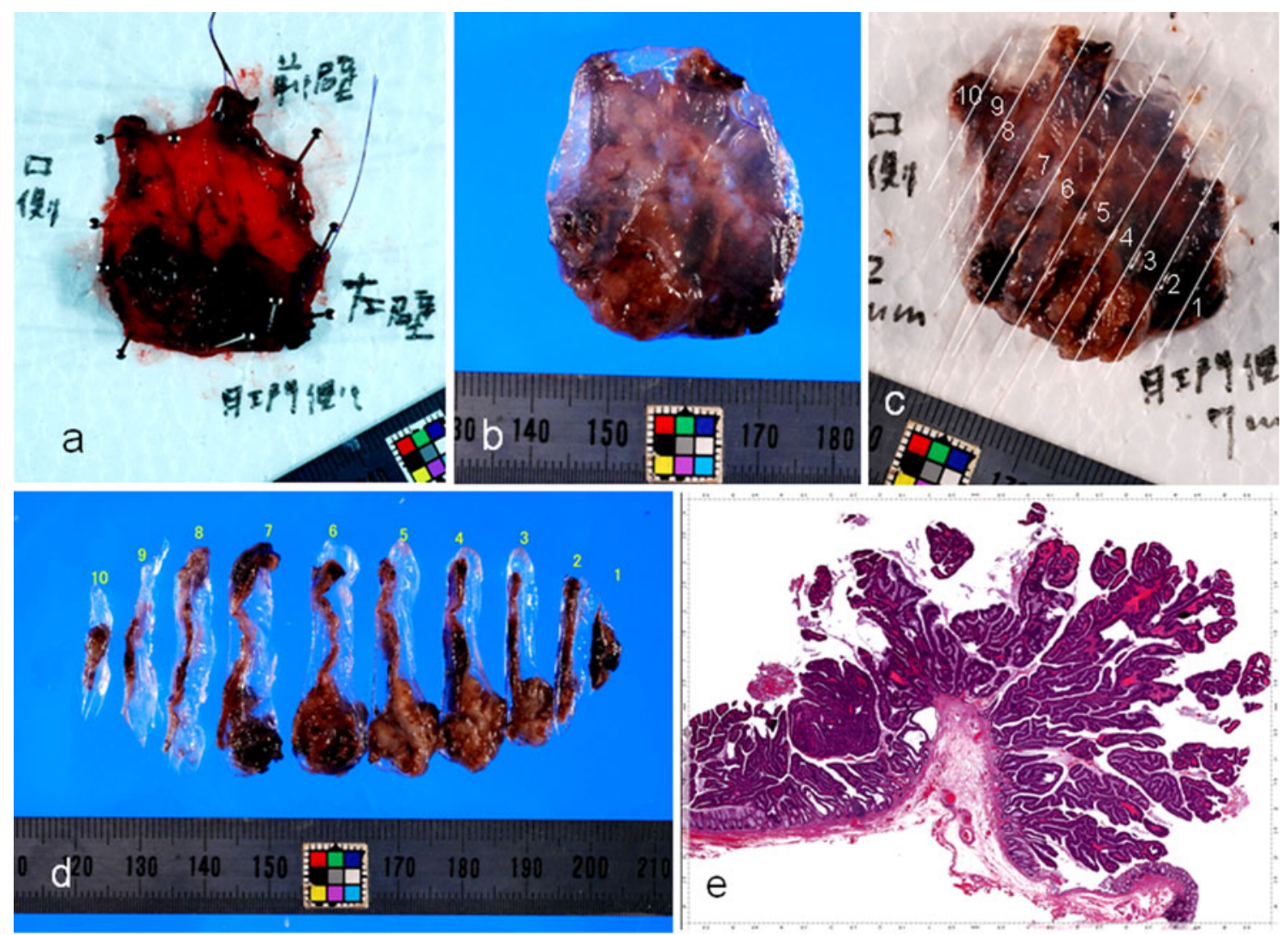

Fig. 1 Tubulovillous adenoma of the rectum treated by endoscopic mucosal resection and examined by the method described in the text. a A piece of mucosa with villous tumor obtained by endoscopic mucosal resection. The specimen was pinned on the expanded polystyrene board and fixed with $10 \%$ formalin. b Endoscopically resected mucosa coated with alginate gel. c The mucosa coated with alginate gel was easily sliced into even parallel sections, each measuring about $3 \mathrm{~mm}$ in thickness. d Ten slices were numbered consecutively. Note that the mucosa is irregular in thickness and villous structures are kept in the alginate gel. e Histological section of the slice no. 4 showed villotubular adenoma. The nearest margin width was $3 \mathrm{~mm}$. The mucus-like substance over the polyp was gelled alginate

\section{References}

slicing has several advantages: (1) villous structures are kept in situ after slicing, (2) the fragile portions are protected from breaking into pieces, and (3) the specimen is given relatively monotonous hard consistency making it easy to get sliced into pieces of even thickness.

This technique is also applicable to the shaved margin evaluation in the breast conservative surgery since the peripheral margins are often irregular and may have crevasses. Sodium alginate itself is structureless material, with faintly purple color in hematoxylin and eosin stain reminiscent of mucin, and does not affect immunostains.

Open Access This article is distributed under the terms of the Creative Commons Attribution Noncommercial License which permits any noncommercial use, distribution, and reproduction in any medium, provided the original author(s) and source are credited.
1. Ohashi K, Momma K, Yamada Y, Yoshida M, Horiguchi S, Matsubayashi J, Shimizu S, Moriyama S, Hishima T, Funata N, Takizawa T, Koike M (2002) Vertical and horizontal growth features of superficial esophageal squamous cell carcinomas: histopathological evaluation of endoscopically resected specimens. Virchows Arch 441:350-357

2. Shiotani A, Haruma K, Uedo N, Iishi H, Ishihara R, Tatsuta M, Kumamoto M, Nakae Y, Ishiguro S, Graham DY (2006) Histological risk markers for non-cardia early gastric cancer. Pattern of mucin expression and gastric cancer. Virchows Arch 449:652-659

3. Ishigami K, Wakasa T, Osawa M, Obatake H, Shiba I, Tanaka S, Nagami Y, Wakasa K (2010) Slicing endoscopically resected mucosa after paraffin embeding (in Japanese). Pathol Clin Med 28:339-342

4. Omori Y, Morichi H, Moriyasu T, Nishimura T, Yamashita K, Zouga O (2008) Specimen preparation for endoscopically resected mucosa using agar (in Japanese). Pathol Clin Med 26:1313-1316

5. Eun SH, Cho JY, Jung IS, Ko BM, Hong SJ, Ryu CB, Kim JO, Jin SY, Lee JS, Lee MS, Shim CS, Kim BS (2007) Effectiveness of sodium alginate as a submucosal injection material for endoscopic mucosal resection in animal. Gut liver 1:27-32 